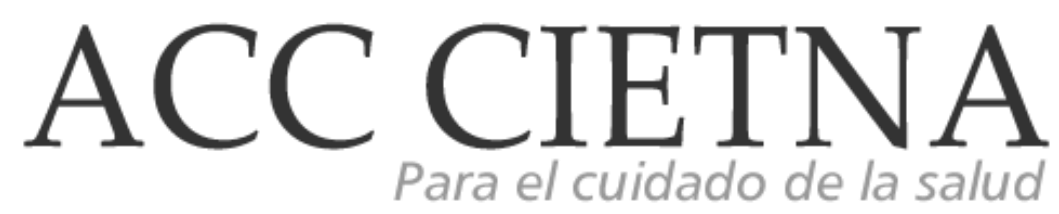

https://doi.org/10.35383/cietna.v7i1.358

\title{
La comunicación y apoyo emocional en enfermería desde la perspectiva del familiar del paciente crítico en un Hospital Público, Chiclayo 2019
}

\section{Gálvez Cerdán Silvia Rocio', Mesta Delgado Rosio del Pilar²}

\begin{tabular}{ll}
\hline INFORMACIÓN DEL ARTÍCULO & RESUMEN \\
\hline Historia del artículo: & Objetivo: Determinar la comunicación verbal, no verbal y apoyo
\end{tabular}

Recibido el 08 de junio de 2020

Aceptado el 28 de junio de 2020

\section{Palabras clave:}

Comunicación (D003142)

Apoyo emocional

Perspectiva del familiar

Enfermería (Q000451)

Crisis situacional
Objetivo: Determinar la comunicación verbal, no verbal y apoyo emocional en enfermería desde la perspectiva del familiar del paciente crítico durante la crisis situacional en un Hospital Público, Chiclayo 2019. Método: Cuantitativo, no experimental, descriptivo de corte trasversal. La muestra fue 93 familiares con parientes directos en estado crítico atendidos en la Unidad de Cuidados Intensivos e Intermedios, y sala de observaciones de Emergencias del Hospital Regional Lambayeque, y el muestreo no probabilístico por conveniencia. Se realizó una prueba piloto con el $25 \%$ de la muestra que obtuvo fiabilidad del $92,6 \%$ de alfa de Cronbach; el procesamiento de datos se realizó en el programa SPSS haciendo análisis estadístico descriptivo, finalmente se practicó criterios éticos universales y rigor científico. Resultados principales: La perspectiva de los familiares sobre comunicación verbal en enfermería fue indiferente (47.3\%); en comunicación no verbal la perspectiva de los familiares resultó indiferente (55.9\%), y, por último, el apoyo emocional tuvo una perspectiva indiferente (66.7\%) seguido de desfavorable (17.2\%). Conclusión general: La comunicación verbal, no verbal y apoyo emocional para el familiar se percibió como indiferente con $47.3 \%, 55,9 \%$ y $17,2 \%$ respectivamente.

1Bachiller de la Escuela de Enfermería de la Universidad Católica Santo Toribio de Mogrovejo, Chiclayo, Perú. email: rocio_24914@hotmail.com. ORCID: https://orcid.org/0000-0002-1511-3898

2Maestra en Enfermería. Docente de la Escuela de Enfermería de Pregrado y II Especialidad de Emergencias y Desastres de la Universidad Católica Santo Toribio de Mogrovejo. Enfermera asistencial del Hospital Nacional Almanzor Aguinaga Asenjo Chiclayo, Perú. email: rmesta@usat.edu.pe. ORCID: https://orcid.org/0000-0003-4635-3170 
The communication and emotional support in nursing since the perspective of familiar of critical patient in a public hospital, Chiclayo 2019

ABSTRACT

\section{Keywords:}

Communication (D003142)

Emotional support

Perspective of family

Nursing (Q000451)

Situational crisis
Objective: Determine the verbal communication, non-verbal communication and emotional support in nursing since the perspective of familiar of the critical patient during the situational crisis in a Public Hospital, Chiclayo 2019. Method: Quantitative, nonexperimental, descriptive of cross-sectional. The sample was 93 familiars with relatives directs in state critical treated in the Intensive and Intermediate Care Unit, and Emergency observation rooms of the Hospital Regional de Lambayeque, and the sampling was nonprobabilistic for convenience. Was carried a to test pilot out with the $25 \%$ of the sample were obtained reliability of $92.6 \%$ of Cronbach's alpha; the data processing was done in the program SPSS doing descriptive statistical analysis, finally were practiced universal ethical criteria and scientific rigor. Main Results: The perspective of the familiars on verbal communication in nursing was indifferent (47.3\%); in non-verbal communication, the perspective of the familiars is indifferent (55.9\%), and, for last, the emotional support had an indifferent perspective (66.7\%) followed by unfavorable (17.2\%). General conclusion: The verbal communication, non-verbal communication and emotional support for the familiar was perceived as indifferent with $47.3 \%, 55,9 \%$ and $17,2 \%$ respectively.

\section{Introducción}

La familia constituye una unidad social, estructural, dinámica y fundamental, que puede ser alterada ante un suceso inesperado como la afectación de la salud de alguno de sus integrantes con tal gravedad que deberá ser internado en áreas de unidades críticas, conllevando al surgimiento de una crisis, desencadenando una serie de necesidades como es el recibir información precisa respecto a la evolución de la salud de su familiar, acercamiento y apoyo emocional integral de parte del profesional de enfermería, así mismo de participación activa del cuidado'.

Travelbee 2,3 sostiene que la tarea del profesional enfermero consiste en asistir y ayudar a la persona y su familia, permitiéndole prevenir y hacer frente cualquier situación de enfermedad y sufrimiento, para ello debe apoyarse en distintas fases frente al encuentro con el familiar, como el acercamiento, reconocimiento de identidades de los miembros del núcleo familiar, nacimiento gradual de empatía, compasión ante la tristeza y desconsuelo, y finalmente al atravesar las fases mencionadas lograr un vínculo que fortifique la relación enfermera-familiar.

La atención de salud del paciente en áreas críticas conformadas por unidades de cuidados intensivos e intermedios, sala de observación de emergencias y recuperación quirúrgica ${ }^{4}$, suele ser tecnicista y el objetivo principal del profesional enfermero está enfocado solo a dar cuidado físico y funcional a la persona enferma, descuidando e incluso olvidando el proceso de afrontamiento en la que sensaciones de ansiedad, estrés, angustia, incertidumbre y depresión son algunos de los sentimientos 
negativos por los que atraviesan los familiares de estas personas, quienes son los principales acompañantes en los procesos de enfermedad 5 .

La comunicación en estas unidades por parte del profesional de enfermería suele ser poco empática y cortante, respuestas breves, e incluso indiferente, expresando gestos de impaciencia, evitando mantener contacto visual y evadiendo las preguntas que el familiar tiene, sin brindar confianza ni apertura para una comunicación fluida ${ }^{6}$, todo esto debido a los diversos procesos administrativos que absorben el tiempo laboral del profesional de enfermería.

Cabe destacar, que, las carencias en habilidades comunicativas en el reporte concedido por el profesional enfermero sobre la evolución de la salud de su familiar, pueden alterar la percepción del familiar y generar conflictos que aumentan la frustración y el estrés; ante ello, es imperioso rescatar las prácticas laborales con competencias y destrezas útiles que favorezcan el surgimiento de una interacción enfermera - familiar de calidad en un ambiente cálido, humanitario y de respeto 7 .

Análogamente, en la configuración de satisfacción de las necesidades, el apoyo emocional integra el soporte percibido por cada familiar de pacientes que están en situación crítica al mantener relación con el profesional enfermero en indicativos como trato cordial, humano, amigable, al mismo tiempo sensaciones de comodidad, confianza y confort. En este sentido, se entiende que el apoyo emocional es un fenómeno que tiene expresiones corporales, de conductas y comportamientos que se visibilizan en la comunicación sea de manera verbal y no verbal, que constituyen elementos básicos que el profesional enfermero debe ejercer ofreciendo apoyo emocional efectivo ${ }^{6}$.

En consecuencia, para esta investigación se propuso la siguiente interrogante: ¿Cuál es la percepción del familiar del paciente crítico respecto a la intervención de enfermería durante la crisis situacional en un Hospital Público, Chiclayo 2019?

\section{Metodología}

La investigación fue no experimental, cuantitativa, descriptiva de corte trasversal ${ }^{8}$. La población lo conformaron 123 familiares de pacientes críticos atendidos en las unidades de cuidados intensivos e intermedios y sala de observación de Emergencia del Hospital Regional Lambayeque. La muestra fue de 93 familiares calculada por el programa estadístico Decision Analyst STATS9; y el muestreo se estableció como no probabilístico por conveniencia. Se incluyó a familiares de pacientes críticos con estancia hospitalaria mínima de 2 días, que sean mayores de edad y familiar directo del paciente crítico; excluyéndose a todo familiar que visitaba por primera vez al paciente crítico.

El instrumento Percepción de los familiares de los pacientes críticos, respecto a la intervención de enfermería durante su crisis situacional, fue diseñado por Franco ${ }^{10}$ y está constituido por 28 enunciados predeterminados tipo Likert; consta de 3 dimensiones: dimensión comunicación verbal, dimensión comunicación no verbal y dimensión apoyo emocional. También contó con confiabilidad y validez de contenido y constructo evaluada por juicio de expertos. Además, se realizó una prueba piloto a 24 familiares y su procesamiento de todos los ítems del instrumento, tuvo como resultado un alfa de Cronbach de $92,6 \%$ confirmando su fiabilidad.

Los enunciados se calificaron del 1 al 5 , en base a proposiciones positivas donde totalmente de acuerdo valía 5 puntos y totalmente desacuerdo 1 punto, y las proposiciones negativas totalmente de acuerdo equivalía 1 punto $y$ totalmente desacuerdo 5 puntos. Se obtuvo los puntajes finales, recodificándolos en 3 categorías: favorable, indiferente y desfavorable; donde los puntajes fueron: Comunicación verbal: favorable 29 a 40 puntos, indiferente 20 a 28 puntos y desfavorable 08 a 19 puntos; 
comunicación no verbal: favorable 40 - 50 puntos, indiferente 29 a 39 puntos y desfavorable 10 a 28 puntos; y apoyo emocional: favorable 24 a 50 puntos, indiferente 17 a 23 puntos y desfavorable 10 a 16 puntos.

En el mes de octubre del 2019 se realizó la recolección de datos a los familiares de pacientes críticos, estableciéndose tiempos y horarios para la aplicación del cuestionario, informándose objetivos, propósitos, beneficios, riesgos $\mathrm{y}$ derechos, así como el consentimiento informado de la investigación. El procesamiento de los datos se hizo tabulándolos en Excel y exportándolos al programa SPSS v.25 para su análisis estadístico descriptivo.

El proyecto recibió aprobación del Comité de Ética de Investigación de la Facultad de Medicina USAT con resolución Nº TRL-2019-12660. Se solicitó permiso al Departamento del Desarrollo de la Investigación Básica Clínica del Hospital Regional Lambayeque para su ejecución, quienes a través de su Comité de Ética concedieron la constancia de aprobación: 0211-044-19, con la cual se hicieron las coordinaciones respectivas con los jefes de los servicios de Unidad de Cuidados Intensivos e Intermedios y sala de observaciones de Emergencias.

La investigadora puso en práctica los principios éticos de Belmont ${ }^{11,12}$ al contacto con los familiares, quienes recibieron respeto de autonomía, condiciones, necesidades y dignidad minimizando daños y maximizando sus beneficios, con el fin de favorecerlos por encima de la investigación, pudiendo suspender la encuesta ante cualquier manifestación de incomodidad, y retomarla sólo si se considera conveniente. Se brindó un trato digno y respetuoso, considerando su vulnerabilidad. De igual importancia, la investigación tuvo evaluación de similitud en el programa anti plagio Turnitin obteniendo un porcentaje de $24 \%$.

\section{Resultados}

\section{TABLA N ${ }^{\circ} 01$}

Perspectiva del familiar del paciente crítico sobre la comunicación verbal, comunicación no verbal y apoyo emocional en enfermería durante la crisis situacional en un Hospital Público, Chiclayo 2019

Percepción Frecuencia Porcentaje

\begin{tabular}{cccc}
\hline \hline \multirow{2}{*}{$\begin{array}{c}\text { Comunicación } \\
\text { Verbal }\end{array}$} & Desfavorable & 30 & $32.3 \%$ \\
& Indiferente & 44 & $47.3 \%$ \\
& Favorable & 19 & $20.4 \%$ \\
\hline \hline \multirow{2}{*}{$\begin{array}{c}\text { Comunicación } \\
\text { no verbal }\end{array}$} & Desfavorable & 93 & $100.0 \%$ \\
\cline { 2 - 3 } & Indiferente & 52 & $20.5 \%$ \\
\hline \hline \multirow{2}{*}{ Apoyo } & Favorable & 21 & $22.6 \%$ \\
\hline Emocional & Total & 93 & $100.0 \%$ \\
\hline & Desfavorable & 16 & $17.2 \%$ \\
& Favorable & 15 & $16.1 \%$ \\
\hline \hline
\end{tabular}

Fuente: Elaboración propia

\section{Interpretación:}

La perspectiva de los familiares sobre la comunicación verbal en enfermería fue indiferente con $47.3 \%$; en la comunicación no verbal la perspectiva corresponde a que para el $55.9 \%$ de familiares es indiferente, y, por último, el apoyo emocional tuvo una perspectiva indiferente del $66.7 \%$ seguido de desfavorable con $17.2 \%$.

\section{Discusión}

La hospitalización en unidades críticas implica el surgimiento de múltiples necesidades no sólo informativas sino afectivas donde los familiares que atraviesan de una vida aparentemente sin preocupaciones, aflicciones o inquietudes en su círculo familiar pasan a un mundo lleno de incertidumbre, nerviosismo, angustia, 
desesperación y sentimientos negativos como consecuencia de la enfermedad de uno de sus integrantes más directos ${ }^{10}$ que según la Ley $\mathrm{N}^{\circ}$ 30012 en el art. 02 todo familiar directo es aquel ya sea hijo, independiente de la edad, padre o madre, conyugue o conviviente ${ }^{13}$, en este sentido, el profesional de enfermería se convierte en el contacto más cercano del familiar, quien en base a su formación profesional recibió la instrucción necesaria para dar cuidado holístico al paciente enfermo $y$ al familiar mediante un trato empático, de comunicación activa, efectiva y asertiva 6 .

De los datos generales de la investigación se obtuvo que la mayoría de personas familiares encargadas de velar por el paciente crítico corresponden al sexo femenino quienes se encuentran muchas veces apostadas fuera de los servicios en espera de alguna información relacionada a la evolución del paciente. Según grupo etario clasificado por el Minsa ${ }^{14}$ en jóvenes entre edades de 18 y 29 años, adultos que oscilan entre los 30 y 59 años, y luego los adultos mayores a partir de 60 años en adelante, en este caso se encontró que en su mayoría son adultos que tienen edades promedio de 30 a 59 años. Respecto, al parentesco con mayor porcentaje fue esposo(a) /conviviente e hijos/hijas ambos con 39,8\%; y por último el tiempo estimado de hospitalización oscila entre los 2 a 6 días con un $78.5 \%$.

Ante la presencia de una crisis situacional, surge consigo desorganización a nivel inter e intrapersonal debido al alejamiento existente con el familiar enfermo, además provoca inestabilidad económica, sobrecarga de trabajo, desorganización en la familia, conflictos y miedo al empeoramiento o perdida repentina del ser querido, muy a parte de la pérdida del sueño y desorden alimenticio por la que atraviesan, ocasionando secuelas a corto y largo plazo en cada uno de ellos ${ }^{15}$.

Así mismo, los familiares desarrollan inseguridad por la evolución de su paciente, debido a la severidad de la enfermedad, ambigüedad de síntomas o la naturaleza de la misma, de tal manera, que la incertidumbre se produce por la débil compresión del propósito y beneficio de los procedimientos $u$ acciones realizadas al paciente ${ }^{16}$. Situaciones donde la interrelación informativa entre enfermera-familiar es débil o escasa, se presenta algunas veces como consecuencia porque el profesional enfermero se encuentra limitado puesto que ésta acción es competencia propia del médico, pero, no quita que la enfermera cuando brinda su cuidado relacionado a su labor de satisfacer las necesidades del paciente - familia dé un cuidado integral interactuando, escuchando y ayudando a aliviar parcialmente el estrés hospitalario que ya tienen ${ }^{17}$.

Sobre la comunicación verbal se evidenció en mayor porcentaje que la perspectiva es indiferente, en puntos como: la enfermera indica sobre procedimientos generales realizados al familiar, usa lenguaje claro y sencillo, las enfermeras no responden en cortésmente ante preguntas de los familiares, llegando a la conclusión que las respuestas son similares a los resultados de la investigación de Franco ${ }^{10}$, contrario a esto son los resultados de Martínez ${ }^{19}$ quien demuestra que la percepción de la comunicación verbal fue desfavorable sobre que la enfermera no enseña forma alguna para el afrontamiento ante sentimientos de angustia, y salta indagar que otras dificultades enfrenta debido a la situación del paciente enfermo.

La comunicación verbal es una herramienta comunicativa que se caracteriza por ser clara, tener vocabulario enriquecido, credibilidad, fluidez, tono adecuado y sencillez al hablar, en la que debe haber respeto de los silencios o pausas, con el fin de facilitar la expresión verbalmente de pensamientos, deseos, creencias, actitudes, sentimientos, percepciones y más, para favorecer la confianza y seguridad de las personas 20 .

Existen situaciones que interfieren en la comunicación verbal y que se dan a causa de 
limitaciones existentes, una de ellas es el tiempo debido a que es mínimo e insuficiente para mantener el contacto cercano con los familiares, y que se relaciona también con la poca colaboración del profesional enfermero, a consecuencia de la falta de formación e instrucción en habilidades comunicativas, usando así un lenguaje técnico que no es entendible o manejado por el familiar, trayendo consigo que ellos se sientan en la necesidad de recurrir a fuentes secundarias de información como internet, que no siempre tiene un respaldo verídico y científico, confundiéndolos aún más; además otra interferencia es el espacio porque las áreas críticas son ambientes de acceso restringido cuyo fin es evitar las sobreinfecciones en los pacientes críticos, atribuyendo negativamente a la relación enfermera familiar $^{21,22}$.

Monsalve, Bancayán y Castro $^{23}$ todos los familiares se encuentran emocionalmente inestables, con temores y dudas, y se recomienda usar la comunicación verbal como instrumento indispensable del cuidado integral para mantener y fortalecer la interrelación enfermera - familiar favorablemente, y poder identificar e intervenir pertinentemente las necesidades comunicativas que puedan surtir.

La comunicación no verbal los familiares investigados reflejaron tener una perspectiva indiferente en indicadores como: la enfermera explica los procedimientos con paciencia, la enfermera tiene mirada cálida al responder inquietudes del familiar, la enfermera dialoga serenamente con los familiares, cuando el familiar pregunta en distintos momentos lo que ya se le explicó la enfermera muestra molestia, y las enfermeras no saludan a los familiares cuando llegan; contrario a Rodríguez y Zuta24 quienes indican que la percepción es favorable. En cuanto a Martinez ${ }^{19}$ sus resultados difieren indicando que la percepción es medianamente favorable, estando a favor cuando la enfermera brinda espacio al familiar para atender sus necesidades $y$ en contra cuando la enfermera muestra gestos que indican molestia por las repetidas preguntas hechas por el familiar.

La comunicación no verbal es una habilidad y el arma más importante del profesional de enfermería, porque su uso impactará considerablemente en beneficio del brindar cuidado de calidad; puesto que su manifestación o exteriorización se realiza a través de gestos como sonrisas, la expresión facial, postura, contacto visual aspectos que ayudan a la evolución de la retroacción entre enfermera familiar, siempre y cuando sean excluidas expresiones inapropiadas como cruzar los brazos mientras se entabla un diálogo, hacer movimientos que muestren impaciencia, exhalaciones bruscas, fruncir el ceño ante las dudas de los familiares, entre otras 23,25 . Todos estos gestos o actitudes que los enfermeros puedan realizar ante algún familiar, por más insignificantes que parezcan podrían limitar el acercamiento, la comunicación y definitivamente romperían ese vínculo importante que hubiera entablado con ellos, por eso es importante que el enfermero tenga la habilidad para no lastimar al familiar y que vele por una buena interacción entre ambos.

El familiar del paciente crítico es un individuo que necesita de alguien que entienda lo que siente, lo reconforte e impulse a no rendirse en su lucha constante y diaria contra el estrés hospitalario, se sugiere que el profesional de enfermería debe escuchar activamente respetando las pausas o silencios del diálogo, mostrando interés y atención en sus expresiones, comprendiendo el difícil momento atravesado mediante gesticulaciones amistosas y cálidas, toque terapéutico, denotando el deseo de ayudar6.

El apoyo emocional obtuvo que un $66,7 \%$ de los familiares de paciente crítico tienen una perspectiva indiferente en momentos como: la enfermera no motiva a la expresión de emociones, toman la mano del familiar al verlos tristes, muestran mínima importancia al estado emocional de los familiares, a diferencia de otra 
investigación hecha en Colombia ${ }^{8}$ que obtuvo el $35,25 \%$ con percepción favorable correspondiente a que el profesional enfermero brinda ánimo al familiar, tranquiliza con palabras que trasmiten aliento y ánimo, y si expresa su preocupación o aflicción el profesional enfermero muestra interés.

Indudablemente el periodo de hospitalización que atraviesa la persona enferma por problemas en su salud, desencadena adicionalmente estrés, ansiedad y alteraciones en el bienestar familiar, dirigiéndolos a apuntar o ver en el profesional enfermero un soporte de ayuda que contemple aspectos biológicos, psicoemocionales y espirituales 26 favoreciendo a la disminución de las perturbaciones emocionales presentes; por tal motivo dentro de las competencias y acciones puestas en práctica debe darse un trato humano, holístico y ver a la persona como un ser con sentimientos y que el manejo de la crisis es distinto entre cada familiar haciéndolos únicos y autónomos 27 .

Es recomendable que el profesional de enfermería tenga como su principal fin a la persona y su todo, ser un pilar de apoyo y compañía que permita la adaptación y manejo de sus habilidades emocionales al familiar del paciente crítico a situaciones difíciles y críticas, brindando una atención humana y de calidad en todas las dimensiones tanto físicas, emocionales y espirituales ${ }^{18}$, suprimiendo el trato al familiar y/o paciente como un número de cama o patología, porque ellos tienen derecho a ser tratados con respeto, dignidad y así mismo el recibir información suficiente y oportuna sobre lo que su familiar enfermo pueda necesitar o cuando este sea sometido a un procedimiento ya sea de diagnóstico o tratamiento.

El apoyo emocional al familiar le proporciona un estado estable y de control, haciéndolo sentirse positivo, seguro, bien en el ambiente que se encuentra, motivándolo a continuar en la lucha de mejora por su familiar enfermo, así como el mantenerlo tranquilo consigo mismo, equilibrando su condición biopsicoemocional 28 . El profesional enfermero debe mostrar interés en los manifiestos de las personas, de ello se evaluará la percepción y necesidades presenten en la familia, estableciendo un plan de cuidados específicos para cada momento, expresando amabilidad, empatía, trato ameno, cálido y empático.

\section{Conclusiones}

La perspectiva en la mayoría de familiares que tienen un pariente directo en situación crítica fue indiferente sobre el actuar de cada profesional enfermero ante una crisis situacional, permitiendo reconocer que, el cuidado brindado tendría que ser holístico, y debería darse más énfasis e importancia a la interacción que tiene la enfermera con el familiar para que ésta se efectué satisfactoriamente haciendo uso esencial de determinados elementos como son la comunicación verbal, comunicación no verbal y apoyo emocional.

La comunicación verbal en enfermería resultó indiferente, en aspectos como la formación de diálogos de origen informativo, en la falta de fluidez y sencillez de la expresión verbal, en el uso de silencios o pausas intermediarias para la manifestación de sentimientos y emociones, todo esto generado por las implicancias del proceso de crisis, en la que está inmerso el profesional enfermero quien cumple el rol de emisor y receptor.

Los familiares de pacientes críticos tuvieron una perspectiva indiferente en la comunicación no verbal, correspondiente a la expresión de gestos, mantenimiento de contacto visual y el uso de contacto físico a través del toque terapéutico, lo que constituye un punto de reflexión para el profesional de enfermería en su labor diaria porque así se garantizaría favorecer un ambiente de cuidado que involucre o integre al familiar.

El apoyo emocional como la dimensión relacionada al entorno psicoafectivo, refleja un 
porcentaje elevado de $66,7 \%$ de perspectiva indiferente seguido de desfavorable, en cuanto al trato ameno, afectivo, atento, amigable y sincero que otorga el profesional enfermero, significando así que el familiar otorga un valor importante al enfoque humanitario y sensible que caracteriza a la enfermera cuando hay presencia de cualquier crisis situacional.

\section{Bibliografía}

1. Boada L. Información: La principal necesidad de las Familias en Unidad de Cuidados Intensivos (UCl) [Tesis de segunda especialidad en Internet]. Colombia: Universidad Nacional de Colombia; 2016 [consultado 13 abril 2019]. 112 p. Disponible en: http://bdigital.unal.edu.co/55973/7/Laura C.BoadaQuijano.2016.pdf

2. Marriner-Tomey A, Raile M. Modelos y Teorías en Enfermería. 8a ed. España: Editorial Elsevier; 2014. 797 p.

3. Kérouac S, Pepin J, Ducharme F, Duquette A, Major F. El pensamiento enfermero. Barcelona: Masson; 1996. 167 p.

4. Lara B, Cataldo A, Castro R, Aguilera P, Ruiz $C$, Andresen M. Medicina de Urgencia y unidades de cuidados intensivos. Una alianza necesaria en busca de la mejoría de la atención de pacientes críticos. Rev Med Chile [Internet]. 2016 [consultado el 24 mayo 2019]; 144: 917 - 24. Disponible en: http://dx.doi.org/10.4067/S003498872016000700014

5. Pinto N. Cuidado y practica de enfermería. Colombia: Editorial Guadalupe Ltda; 2006. $271 \mathrm{p}$.

6. Bautista L, Arias M, Carreño Z. Percepción de los familiares de pacientes críticos hospitalizados respecto a la comunicación y apoyo emocional. Rev Cuid [Internet]. 2016 [consultado 12 abril 2019]; 7 (2): 1297 $1309 . \quad$ Disponible en: https://doi.org/10.15649/cuidarte.v7i2.33 0
7. Heras G, Cruz M, Nin N. Buscando humanizar los cuidados intensivos. Rev Bras Ter Intensiva [Internet]. 2017 [consultado 12 abril 2019]; 29 (1): 9 - 1. Disponible en: https://www.scielo.br/scielo.php?script=sci _arttext\&pid=S0103-507X2017000100009

8. Hernández-Sampieri R, Mendoza C. Metodología de la investigación: las rutas cuantitativa, cualitativa y mixta. $2^{\text {da }}$ ed. México: Mc Graw Hill; 2018. 754 p.

9. Decision Analyst. Software Decision Analyst [Internet]. Canadá: STATS; 2018 [consultado 26 junio 2019]. Disponible en: https://www.decisionanalyst.com/download I

10. Franco R. Percepción del familiar del paciente crítico, respecto a la intervención de enfermería durante la crisis situacional en la Unidad de Cuidados Intensivos. Hospital Nacional Edgardo Rebagliati M., abril 2003 [Tesis de licenciatura en Internet]. Perú: Universidad Nacional Mayor de San Marcos; 2003 [consultado el 30 de marzo 2019]. 53 p. Disponible en: https://url2.cl/xv56i

11. Office for Human Research Protections. The Belmont Report. OHRP [Internet]. 2018 [consultado 31 mayo 2019]. Disponible en: https://url2.cl/c4uDL

12. Morales J, Nava G, Esquivel J, Díaz L. Principios de ética, bioética y conocimiento del hombre. México: Universidad Autónoma de Hidalgo; 2011.294 p.

13. El peruano. Aprueban Reglamento de la Ley $N^{\circ} 30012$, Ley que concede el derecho de licencia a trabajadores con familiares directos que se encuentran con enfermedades en estado grave o terminal o sufran accidente grave [Internet]. Perú: Diario Oficial del Bicentenario; 2017 [consultado 26 de octubre 2019]. Disponible en: https://url2.cl/vcWCm

14. MINSA. Perú: Porcentaje de población por etapas de vida. [Internet]. Perú: Indicadores de Salud; 2016 [consultado 26 de octubre 2019]. Disponible en: https://url2.cl/SPKy3 
15. Puggina A, Begosso K, Carbonari F, Stach L, Sapatini $T$ et al. Percepção da comunicação, satisfação e necessidades dos familiares em Unidade de Terapia Intensiva. Escola Anna Nery Revista de Enfermagem [Internet]. 2014 [consultado 07 noviembre 2019]; 18 (2): 277-283. Disponible en: http://www.scielo.br/pdf/ean/v18n2/1414 -8145-ean-18-02-0277.pdf

16. Flórez I, Montalvo A, Romero E. Incertidumbre en cuidadores familiares de pacientes hospitalizados en unidades de cuidado intensivo. Investigación en enfermería [Internet]. 2018 [consultado 05 de noviembre 2019]; 20 (1): 2027 - 128. Disponible:

https://doi.org/10.11144/Javeriana.ie20$1 . i c f p$

17. Sánchez-Vallejo A. Fernández D. PérezGutiérrez A y Fernández-Fernández $M$. Análisis de las necesidades de la familia del paciente crítico y la opinión de los profesionales de la unidad de cuidados intensivos. Elsevier: Medicina Intensiva [Internet]. 2016 [consultado 10 de noviembre 2019]; 40 (9): 527 - 540. Disponible en: http://dx.doi.org/10.1016/j.medin.2016.0 3.005

18. Lujan J, Díaz J, Paz M, Dziad L, Duarte L y Gómez C. Percepción de la familia del paciente crítico sobre enfermería, en el horario de visita en un Hospital de Alta Complejidad. Revista del Hospital El Cruce [Internet]. 2017 [consultado 07 de noviembre 2019]; 7 (21): 16 - 23. Disponible en: https://url2.cl/2qqBe

19. Martínez K. Percepción del familiar del paciente crítico, sobre la atención que brinda la enfermera ante una crisis situacional en los servicios de medicina de un Hospital Nacional de Lima - 2015 [Tesis de licenciatura en Internet]. Perú: Universidad Nacional Mayor de San Marcos; 2015 [consultado el 11 de mayo 2019]. 95 p. Disponible en: https://url2.cl/4CLTk
20. García E, Gil F, Huamani A. Percepción de los familiares del paciente respecto a la comunicación con la enfermera en el servicio crítico del cuidado de la mujer del "Hospital Nacional Docente Madre Niño San Bartolomé", Lima 2018. [Tesis de segunda especialidad en Internet]. Perú: Universidad Peruana Cayetano Heredia; 2018 [consultado 11 de mayo 2019]. 44 p. Disponible en: https://url2.cl/NLGIH

21. Camargo L, Taipe V, Chero V. Percepción de los padres y calidad en el cuidado de enfermería a pacientes pediátricos en un hospital en Perú, 2017. Ágora Rev. Cient [Internet]. 2017 [consultado 23 febrero 2020]; 4 (2): e3. Disponible en: https://url2.cl/uHVNI

22. Achury D, Pinilla M, Alvarado $H$. Aspects that facilitate or interfere in the communication process between nursing professionals and patients in critical state. Investigación y Educación en Enfermería [Internet]. 2015 [consultado el 20 febrero 2020]; 33 (1): 102 - 111 Disponible en: http://www.scielo.org.co/scielo.php?script= sci_arttext\&pid=S0120$53072015000100012 \& \operatorname{lng}=$ es\&nrm $=$ iso

23. Monsalve M, Bancayán S, Castro R. La comunicación enfermera - persona cuidada hospitalizada en el servicio de oncología en un hospital de Essalud, Chiclayo 2017 [Tesis de licenciatura en Internet]. Lambayeque: Universidad Pedro Ruiz Gallo; 2017 [consultado 15 de febrero 2020]. 107 p. Disponible en: https://url2.cl/fNHWs

24. Rodríguez M, Zuta R. "Nivel de satisfacción del familiar del paciente respecto a la comunicación brindada por la enfermera de cuidados intensivos hospital nacional Carlos Alberto Seguín Escobedo - 2016" [Tesis de segunda especialidad en Internet]. Arequipa: Universidad Nacional de San Agustín de Arequipa; 2017 [consultado el 15 de febrero 2020]. 103 p. Disponible en: http:// repositorio.unsa.edu.pe/bitstream/h 
andle/UNSA/4606/ENSroyamm.pdf?sequenc

$\mathrm{e}=1$ \&isAllowed $=\mathrm{y}$

25. CODEM. Comunicación efectiva enfermerapaciente: Una estrategia más en el cuidado [Internet]. España: Colegio Oficial de Enfermería de Madrid; 2015 [consultado 20 de febrero 2020]. Disponible en: https://www.codem.es/noticias/comunicaci on-efectiva-enfermera-paciente-unaestrategia-mas-en-cuidado

26. Silva J, Ramón S, Vergaray S, Palacios V, Partezai R. Percepción del paciente hospitalizado respecto a la atención de enfermería en un hospital público [Internet]. 2015 [consult ado 22 febrero 2020]; 12 (2): 80-87. Disponible en: https://doi.org/10.1016/j.reu.2015.04.001

27. Landman C, Cruz M, García E, Pérez P, Sandoval P, Serey K, Valdés C. Satisfacción usuaria respecto a competencia de comunicación del profesional de enfermería. Ciencia y enfermería [Internet]. 2015 [consultado el 01 de marzo 2020]; 21 (1): 91 - $102 . \quad$ Disponible en: https://scielo.conicyt.cl/pdf/cienf/v2 ln1/ar t_09.pdf

28. Castañon C. Percepción familiar sobre cuidado y apoyo emocional que brinda el enfermero al usuario del servicio de medicina del Hospital Regional Hermilio Valdizan Medrano, Huánuco 2016 [Tesis de licenciatura en Internet]. Huánuco: Universidad de Huánuco; 2017 [consultado 6 marzo 2020]. 114 p. Disponible en: https://url2.cl/W9DcZ 